\title{
Pancake-like and tablet-like distribution functions of energetic electrons in the middle magnetosphere
}

\author{
Peter Bespalov ${ }^{1}$ and Olga Savina ${ }^{2}$ \\ ${ }^{1}$ Institute of Applied Physics RAS, Nizhniy Novgorod, Russia \\ ${ }^{2}$ National Research University Higher School of Economics, Nizhniy Novgorod, Russia \\ Correspondence to: Peter Bespalov (pbespalov@mail.ru)
}

Received: 3 December 2016 - Revised: 29 December 2016 - Accepted: 3 January 2017 - Published: 24 January 2017

\begin{abstract}
We propose a simple explanation of the prolonged existence of pancake-like electron velocity distributions in the radiation belts. The pancake-like distribution function is characterized by a longitudinal particle velocity (along the magnetic field) of the order of the thermal velocity of the background plasma. The parameters of the tablet-like distribution function with a characteristic longitudinal particle velocity of the order of 20 Alfvèn velocities are refined. Such distribution functions can occur in the middle magnetosphere near the magnetic equator with appropriate sources of energetic particles. The stability of these distributions is examined. The results agree with known experimental data.
\end{abstract}

Keywords. Magnetospheric physics (energetic particles trapped)

\section{Introduction}

A few years ago, great attention was paid to the analysis of the evolution of the Earth's electron radiation belts (RBs) taking into account both the particle radial diffusion across the magnetic shells and the quasi-linear relaxation effects of the energetic-particle distribution function due to the interaction with whistler waves (e.g., Bespalov and Trakhtengerts, 1986). Usually, while performing calculations, the original system of quasi-linear equations was averaged over the bounce period of charged particle oscillations between the mirror points for smooth distribution functions over the equatorial pitch angle. Recently, important studies in this direction were carried out to explain new experimental data and for a quantitative simulation of the RB dynamics (Thorne et al., 2013; Yang et al., 2016; Su et al., 2016; Albert et al., 2016). Energetic electrons with a smooth distribution func- tion may be responsible for the excitation of whistler electromagnetic emission with fine spectral forms (e.g., Manninen et al., 2014). In all the cases, the possibility of the existence of particle fractions with sharp (possibly, non-analytical) distribution functions was not specially examined in the analysis of the averaged RB dynamics.

In actual fact, in the RB there are different factors determining the sources and losses of particles in the plasma magnetic trap. Therefore, rather different particle distribution functions can exist for a short time in the velocity space. On the contrary, the protractedly existing distributions coordinated with the processes in the plasma magnetic trap are stable enough. Keeping in mind the recently reached increase in the volume and accuracy of experimental data on the particle distribution functions in the magnetosphere, it is expedient to return to the problem of some protractedly existing sharp distribution functions.

Evidence for the existence of the pancake-like and tabletlike energetic electron distributions in the velocity space (Fig. 1) near the magnetic equator plane in the RB has recently been obtained in the space experiments. Asnes et al. (2005) give experimental data obtained using the Magnetospheric Plasma Analyser (MPA) instrument onboard the LANL geosynchronous satellites, according to which pancake-like distributions of the energetic electrons with energies less than $47 \mathrm{keV}$ can exist. The authors consider such distributions as resulting from the wave-particle interaction with chorus emissions outside the plasmasphere due to the Cherenkov resonance.

Unusual triggered emissions (Bell et al., 2000) were registered on the magnetic shell $L=3.4$ by the POLAR space vehicle, when a distribution function with strong transverse anisotropy was observed in the magnetosphere. This distribu- 

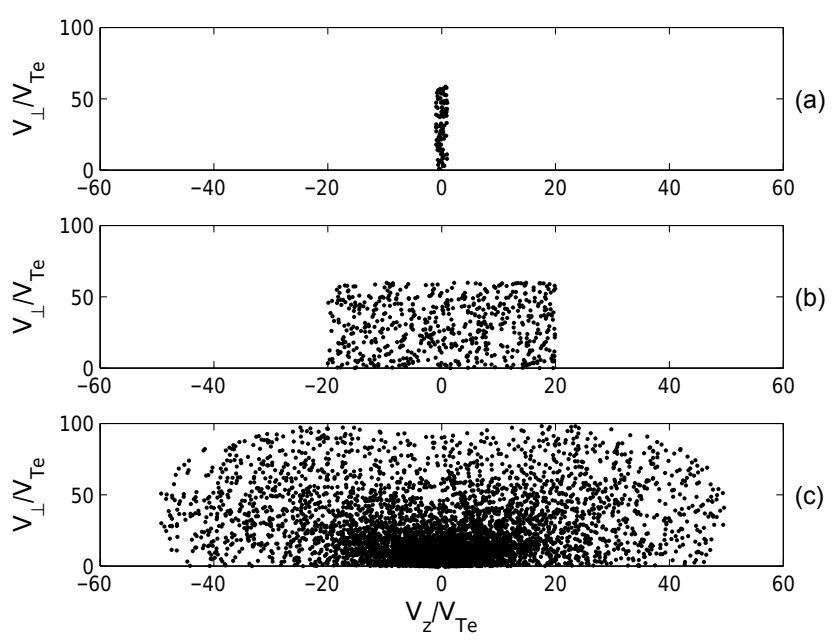

Figure 1. Schematic diagram for pancake-like (a), tablet-like (b), and smooth (c) distributions in the velocity space $\left(V_{\perp}, V_{z}\right)$.

tion function is similar to the tablet-like one since the equatorial pitch angle of particles was about $75^{\circ}$ or more.

Sharp distribution functions were also studied in the theoretical works. The moving step-like distribution function was examined in several publications, beginning with the paper by Bespalov and Trakhtengerts (1986). In paper Trakhtengerts et al. (2001), it was mentioned that the sharp anisotropic distributions can be useful for explaining some properties of the chorus and triggered emissions. The authors of Hikishima et al. (2009) connected the pancake-like distribution with the excitation of chorus emissions in the magnetosphere.

In this paper, we examine special features of the energetic electron populations with pancake-like and tablet-like distribution functions in the middle magnetosphere near the magnetic equator plane. It is shown that the indicated distributions can exist for a long time because of the relatively weak interaction with the wave perturbations determining the dynamics of the bulk of energetic electrons. Stability of these distributions is explored. For definiteness, the electron component at the RB is discussed. Many conclusions are also valid for similar ion distribution functions.

\section{Pancake-like distribution function as specific to the radiation belts}

It is well known that the electron RB dynamics are in many respects determined by interaction of particles with the whistler waves. Such an interaction is effective when the resonance condition is satisfied:

$\omega-k_{z} V_{z}=s \omega_{B} / \gamma_{L}$,

where $\omega$ is the frequency of the wave, $k_{z}$ is the component of the wave vector along the magnetic field $\boldsymbol{B}, \gamma_{L}=(1-$
$\left.V^{2} / c^{2}\right)^{-1 / 2}$ is the Lorentz factor, $V_{z}$ and $V$ are the electron velocity along the magnetic field and their total velocity, $\omega_{B}$ is the value of electron cyclotron frequency (nonrelativistic), $s$ is the number of the cyclotron harmonic, and $c$ is the speed of light.

Let us examine the population of the energetic electrons, for which in the process of their bounce oscillations the following condition is always fulfilled:

$\left|V_{z}\right|<V_{\mathrm{Te}}$

where $V_{\mathrm{Te}}$ is the thermal velocity of background plasma, which is usually of the of order $V_{\mathrm{Te}} \approx 10^{8} \mathrm{~cm} \mathrm{~s}^{-1}$. The electrons with small longitudinal and comparatively high transversal velocities shown in Fig. 1a form the pancake-like distribution function in the plane of the magnetic equator. The common form of this distribution function can conventionally be represented by means of Dirac's $\delta$ function:

$F=n_{L} \frac{f\left(V_{\perp L}\right) \delta\left(V_{z L}\right)}{2 \pi V_{\perp L}}$,

where $n_{L}$ is the density of energetic electrons, $f\left(V_{\perp L}\right)$ is the distribution with respect to the transverse velocities, and the values on the plane of the magnetic equator are written with the index $L$.

The whistler waves, which are able to cause spreading of distribution function (3) with respect to the longitudinal velocity in accordance with Eqs. (1) and (2), also interact with a considerably denser equilibrium plasma. Strong refraction and damping in the equilibrium background plasma prevent the propagation of the right-hand polarized radiation towards the cyclotron resonance region (Gospodchikov, 2007). Therefore, the corresponding waves are characterized by low intensity. The low intensity of the resonance whistler waves determines the possibility of the prolonged existence of the pancake-like distribution function (3).

Preliminary conclusions can be drawn about the range of transverse velocities in the distribution function (3). For relatively low transverse velocities, the Coulomb collisions are important and can ensure the isotropic form of the distribution function. It is possible to estimate a typical particle lifetime in the distribution function (3) with the transverse velocity $V_{\min }$ by means of the relationship $\tau \simeq\left(1 / v_{e}\right)\left(V_{\min } / V_{\mathrm{Te}}\right)^{3}$, where $v_{e}$ is the frequency of Coulomb collisions, which depends on the background plasma density $n_{\mathrm{p}}$, thermal velocity $V_{\mathrm{Te}}$, and Coulomb logarithm. Therefore,

$V_{\min } \simeq V_{\mathrm{Te}}\left(\tau v_{e}\right)^{1 / 3}$.

Let us estimate now a top value of the transverse velocities in the distribution (3). At relatively high transverse velocities, in Eq. (1) it is necessary to take into account relativistic correction to the gyrofrequency $\omega_{B}$. In this case, the pitch-angle particle scattering by the waves which do not attenuate in the background plasma becomes possible. The maximum transverse velocity in the pancake-like distribution corresponds 
to the condition under which the Doppler correction to the frequency is compared with the relativistic correction to the gyrofrequency

$$
\frac{|s| \omega_{B} V_{\max }^{2}}{2 c^{2}} \simeq\left|k_{z \max } V_{\mathrm{Te}}\right|
$$

For the whistler waves which interact with the electron RB in the Earth's magnetosphere, the parameter $k_{\perp} V_{\perp} / \omega_{B}<1$ and main wave-particle interaction usually occur at the cyclotron harmonic with $s=1$. As an estimation of the maximum value of the wave vector longitudinal component, it is possible to take $k_{z \max } \simeq \omega_{\mathrm{pL}} / c$, which corresponds in the longitudinal propagation of whistler waves $\left(\left|k_{z} / k\right| \approx 1\right)$ to the frequency $\omega \simeq \omega_{B L} / 2$. Then relationship (5) is reduced to the form

$$
V_{\max } \simeq\left(2 V_{\mathrm{Te}} c \omega_{\mathrm{pL}} / \omega_{B L}\right)^{1 / 2},
$$

which confirms the nonrelativistic status of the distribution function (3).

Particles with the pancake-like distribution function in the mentioned range of transverse velocities in the equatorial magnetosphere are located in the "shadow" of the background plasma. If there are noticeable sources of such particles, then their accumulation will occur. This particle population is weakly connected with others. It is important to note that the possibility of the prolonged existence of distribution function (3) follows from the general resonance condition (Eq. 1), and it is not connected with the concrete wave mode type.

\section{Tablet-like distribution function beyond the boundary of particle diffusion in the velocity space}

Other particle distributions with a considerably greater spread along the longitudinal velocity were also obtained in the RB (Bell et al., 2000; Asnes et al., 2005). Actually, whistler waves exist in a certain frequency band, which is determined by the mean distribution function anisotropy, frequency dependence of the magnetospheric resonator quality, and special features of the wave ray tracing. Because of the integral nature of the wave-particle interaction in an inhomogeneous magnetic trap, the case is possible where not all the particles supplied by the source interact with the whistler waves. The absence of the local one-to-one connection of the frequency band with longitudinal velocity of energetic electrons in the magnetic equator plane specifies the existence of the minimum longitudinal velocity of the particles which participate in the interaction with the waves. Therefore, in the region of low longitudinal velocities, the accumulation of particles is possible, and a tablet-like distribution, which differs from the pancake-like one by a larger spread along the longitudinal velocity, may occur.

To explain this assertion, we assume that in the magnetic field tube at the moment $t=0$, the source of particles with the power

$J=C \mu^{q} \exp \left(-V^{2} / V_{0}^{2}\right)$,

starts to act depending on the first adiabatic invariant $\mu=$ $m V_{\perp}^{2} / 2 B$ and the square of the total velocity $V^{2}=V_{z}^{2}+$ $2 \mu B / m$, where $V_{0}^{2}$ is square of the characteristic velocity, $m$ is the mass of electron, and $C$ and $q$ are positive constants.

In the presence of the source, the particle distribution function in the magnetic field tube will increase proportionally with time; if it is not corrected by the processes of the pitchangle scattering by the whistler waves,

$F=J t$

Distribution function (8) determines the local distribution function in the velocity space at any point along the magnetic field. If whistler waves with frequency $\omega$ exist in the inhomogeneous magnetic field tube, then in order to explain what particles and where these waves interact, it is necessary to write down the cyclotron resonance condition and the dispersion equation. In the case of longitudinal propagation $\left(\left|k_{z} / k\right| \approx 1\right)$ and of a refractive index considerably greater than 1 , these relationships take the following form:

$\omega-k V_{z}=\omega_{B}$,

$\left(\frac{k c}{\omega}\right)^{2}=\frac{\omega_{p}^{2}}{\omega\left(\omega_{B}-\omega\right)}$.

For the distribution function (8) it is not difficult to determine the total amplification $\Gamma=\int_{-l / 2}^{l / 2} 2\left(\gamma / V_{\mathrm{g}}\right) \mathrm{d} z$ of whistler waves due to the cyclotron instability during the single wave passage between the conjugate ionospheric regions, where $l$ is the length of the magnetic field tube, $\gamma$ is the increment of cyclotron instability, $V_{\mathrm{g}}$ is the group velocity of whistler waves, and $z$ is the coordinate along the magnetic field line measured from the magnetic equator. Calculations show that

$$
\begin{aligned}
\Gamma= & A\left[\frac{\omega}{\omega_{B L}}\left(2+\frac{\omega}{\omega_{B L}}\right)\right]^{-1 / 2}\left[q-(q+1) \frac{\omega}{\omega_{B L}}\right] \\
& \times \exp \left[-\frac{\omega_{B L}}{\beta_{*} \omega}\left(1-\frac{\omega_{B L}}{\omega}\right)^{2}\right] .
\end{aligned}
$$

Here, $A=\sqrt{2} \pi n_{L} L R_{\mathrm{E}} \omega_{\mathrm{pL}} / 3 n_{\mathrm{pL}} c$, where $L$ is the parameter of magnetic shell, $R_{\mathrm{E}}$ is the radius of the Earth, and $\beta_{*}=$ $\left(\omega_{\mathrm{pL}} V_{0} / \omega_{B L} c\right)^{2}$. The dependence of the total amplification on the frequency and energy of particles is given in Fig. 2. The total amplification (10) reverses sign at the frequency

$\omega_{\max }=\frac{q}{q+1} \omega_{B L}$.

Let us note that similar to Eq. (10), frequency dependence also occurs for the maximum at the angle between the wave 


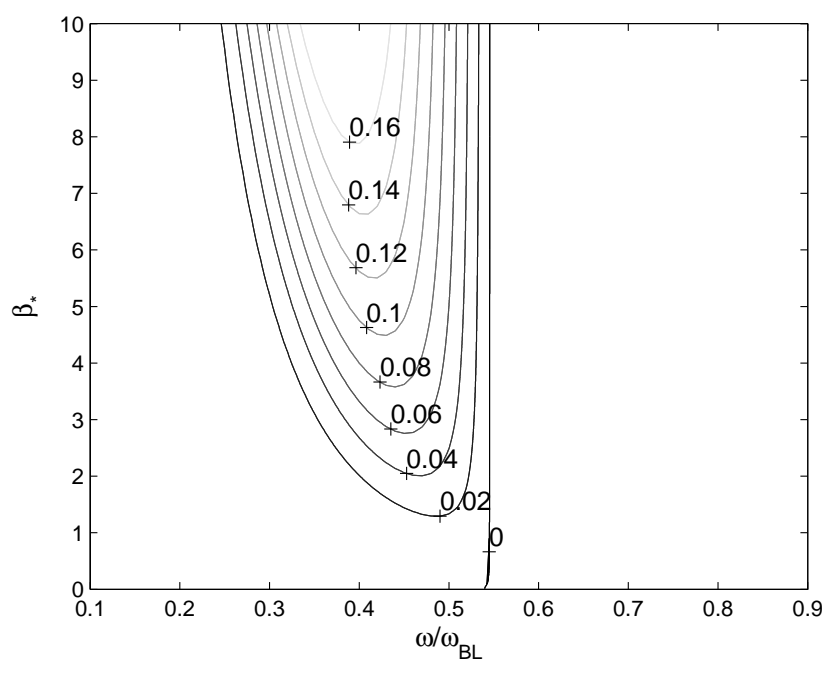

Figure 2. Dependence of the relative total amplification $\Gamma / A$ on the relative frequency $\omega / \omega_{B L}$ and the energy of particles, characterized by the parameter $\beta_{*}$, with $q=1.2$.

vector and the magnetic field of increment on the top of the magnetic field tube (Schriver et al., 2010).

In obtaining Eq. (10) for the total amplification, it was assumed that the distribution of the plasma density along the magnetic field near its top corresponds to the condition: $\omega_{p}^{2} / \omega_{B}=\omega_{\mathrm{pL}}^{2} / \omega_{B L}$. This relationship qualitatively agrees with the experimental data and the results of calculations in the model of the diffusive equilibrium (Persoon et al., 2009). Excluding from Eqs. (9) the wave number and assuming for simplicity the accepted condition of the density distribution, we obtain

$\frac{\left(\omega_{B} / \omega-1\right)^{3}}{\left(\omega_{B} / \omega\right)}=\frac{\omega_{\mathrm{pL}}^{2} V_{z}^{2}}{\omega \omega_{B L} c^{2}}$.

Moving away from the equator the left-hand side of Eq. (12) increases (with increasing of $\omega_{B}(z)$ ), while the right-hand side decreases (with decreasing of $V_{z}^{2}(z)$ ). If $V_{z L}^{2}$ is sufficiently large, then for the selected frequency $\omega$, there are two symmetrically arranged points of wave-particle interaction outside of the magnetic equator, whose precise position can be found by solving the algebraic equation. If $V_{z L}^{2}$ is not so large, then there will be one point of interaction at the magnetic equator, and for even smaller $V_{z L}^{2}$ this point disappears, too. Therefore, there is a minimum value of $V_{z L}^{2}$, which corresponds to the boundary of the wave-particle interaction in the magnetic equatorial plane even at the maximum possible frequency (11) (Goldstein and Tsurutani, 1984; Bespalov and Trakhtengerts, 1986; Asnes et al., 2005):

$\frac{\left(\omega_{B L}-\omega_{\max }\right)^{3}}{\left(\omega_{\max } \omega_{B L}^{2}\right)}=\left(\frac{\omega_{\mathrm{pL}} V_{z L \min }}{\omega_{B L} c}\right)^{2}$.

For a relatively small anisotropy of the particle source power $q \simeq 1$, following relationship (11) we have $\omega_{\max } \simeq \omega_{B L} / 2$.
Therefore, in accordance with Eq. (13), we find

$V_{z L \min }=\frac{c \omega_{B L}}{2 \omega_{\mathrm{pL}}} \simeq 21 \times V_{\mathrm{A}}$

where $V_{\mathrm{A}}$ is the Alfvèn velocity if the ions are predominantly protons. Therefore, inside the plasmasphere, the minimum longitudinal velocity is considerably less than outside. Characteristic velocity (14) is equal to $V_{z L \min } \approx 2 \times 10^{9} \mathrm{~cm} \mathrm{~s}^{-1}$.

If in the magnetic field tube there are electron sources with such or lower longitudinal velocities, then these particles will be accumulated in significant quantities without participating in the cyclotron wave-particle interaction. Therefore, a tablet-like distribution function (see Fig. 1b) can be formed in the equatorial magnetosphere.

\section{Instability of plasma with distribution functions in question}

The question regarding instabilities of the particle distributions with the pancake-like and tablet-like distributions has been examined in sufficient detail in the literature (e.g., Zhelezniakov and Zlotnik, 1989) in connection with the astrophysical applications. On the one hand, such distributions simulate rather well conditions in a strongly nonequilibrium plasma. On the other hand, their use considerably simplifies computations, which is completely justified when there are no precise data about the real distribution function of the charged particles. The pancake-like and tablet-like distributions can be unstably relative to the excitation of electrostatic oscillations at half-integer electron cyclotron harmonics $(\mathrm{ECH}) \omega=(s+1 / 2) \omega_{B}$ with the wave vectors across the magnetic field. Such oscillations exist in the daytime magnetosphere beyond the plasmapause (Christiansen et al., 1978). The greatest possible increment of the corresponding instability is realized at the dual plasma resonance (Zhelezniakov and Zlotnik, 1989) when the frequency of the electrostatic oscillations is equal to

$s \omega_{B L} \simeq\left(\omega_{\mathrm{pL}}^{2}+\omega_{B L}^{2}\right)^{1 / 2}$.

In actual fact, these relatively high-frequency oscillations can be excited due to the positive derivative of the transverse velocity distribution $\left(\partial f / \partial V_{\perp}>0\right)$. The maximum increment of instability is close to

$\gamma \simeq \omega_{B L} \frac{n_{L}}{n_{\mathrm{pL}}}$.

Instability with such a large increment ensures rapid spreading of the transverse velocity distribution function and the formation of the distribution decay to the high velocities. One should note that there are no instabilities of this type in relatively dense cold background plasma when the existence of the slowly decaying electrostatic oscillations (ECHs) is not possible. 


\section{Conclusions}

It is not only smooth distribution functions of the energetic electrons at the pitch angles but also sharp ones that can exist in the electron radiation belts which effectively interact with the whistler electromagnetic waves. In this paper, two of them are examined, namely, the pancake-like and tabletlike particle distributions in the velocity space.

The formation and prolonged existence of the pancake-like distribution functions of energetic electrons are possible in the equatorial region of the plasma magnetic trap. The longitudinal velocity of electrons in this distribution is of the order of the thermal velocity of the background plasma. The energies of particles in this distribution range from epithermal to significant but not relativistic.

In the same region of space, a tablet-like distribution is also possible. As is known, this distribution corresponds to the special features of the interaction of whistler waves with energetic electrons. This interaction is not effective for relatively small longitudinal velocities, i.e., lower than 20 Alfvèn velocities.

Note that the question about the specific properties of the pancake-like and tablet-like distribution can be addressed from somewhat different positions, for example, by examining the coefficient of diffusion of particles in the velocity space. If we turn to the results of such quasi-linear calculations, these then explain why, in the region of small longitudinal velocities (along the magnetic field), there is no diffusion coefficient at the longitudinal velocities for all basic types of the magnetospheric electromagnetic disturbances.

The possibility of the existence of pancake-like or tabletlike distribution functions of energetic particles in the magnetosphere is determined by the properties of the sources of particles with small longitudinal velocities in the equatorial region of the radiation belts. For example, substorm particle injection, particle radial diffusion, and decay of secondary neutrons, which appear in the internal magnetosphere as a result of charge-exchange collisions, can serve as such sources.

Distributions of the examined type can be a reason for the excitation of electrostatic cyclotron emissions at the halfinteger harmonics in the daytime magnetosphere.

Competing interests. The authors declare that they have no conflict of interest.

Acknowledgements. P. Bespalov was supported by RSF Grant no. 16-12-10528.

The topical editor, E. Roussos, thanks one anonymous referee for help in evaluating this paper.

\section{References}

Albert, J. M., Starks, M. J., Horne, R. B., Meredith, N. P., and Glauert, S. A.: Quasi-linear simulations of inner radiation belt electron pitch angle and energy distributions, Geophys. Res. Lett., 43, 2381-2388, doi:10.1002/2016GL067938, 2016.

Asnes, A., Stadsnes, J., Friedel, R. W., Ostgaard, N., and Thomsen, M.: Medium energy pitch angle distribution during substorm injected electron clouds, Geophys. Res. Lett., 32, L10101, doi:10.1029/2004GL022008, 2005.

Bell, T. F., Inan U. S., Helliwell, R. A., and Scudder J. D.: Simultaneous triggered VLF emissions and energetic electron distributions observed on POLAR with PWI and HYDRA, Geophys. Res. Lett., 27, 165-168, doi:10.1029/1999GL003673, 2000.

Bespalov, P. A. and Trakhtengerts, V. Y.: Cyclotron instability of the Earth radiation belts, Reviews of Plasma Physics, 10, 155-292, Consultants Bureau, New York, USA, 1986.

Christiansen, P. J., Gough, M. P., Martelli, G., Bloch, J. J., Cornillean, N., Etcheto, J., Gendrin, R., Beghin, C., and Decreau, P.: GEOS-1 observations of electrostatic waves, and their relationship with plasma parameters, Space Sci. Rev., 22, 383-400, doi:10.1007/BF00210875, 1978.

Goldstein, B. E. and Tsurutani, B. T.: Wave normal direction of chorus near the equatorial source region, J. Geophys. Res., 89, 2789-2810, doi:10.1029/JA089iA05p02789, 1984.

Gospodchikov, E. D., Smolyakova, O. B., and Suvorov, E. V.: Ray trajectories and electron cyclotron absorption in an axisymmetric magnetic confinement system, Plasma Phys. Rep., 33, 427-434, doi:10.1134/S1063780X0705008X, 2007.

Hikishima, M., Yagitani, S., Omura, Y., and Nagano, I.: Coherent nonlinear scattering of energetic electrons in the process of whistler mode chorus generation, J. Geophys. Res., 114, A10205, doi:10.1029/2009JA014371, 2009.

Manninen, J., Kleimenova, N. G., Fedorenko, Yu. V., Bespalov, P. A., and Turunen, T.: New results of structured VLF emissions observed simultaneously at two closely located stations near $L \approx 5.5$, Ann. Geophys., 32, 1163-1167, doi:10.5194/angeo-321163-2014, 2014.

Persoon, A. M., Gurnett, D. A., Santolik, O., Kurth, W. S., Faden, J. B., Groene, J. B., Lewis, G. R., Coates, A. J., Wilson, R. J., Tokar, R. L., Wahlund, J.-E., and Moncuquet, M.: A diffusive equilibrium model for the plasma density in Saturn's magnetosphere, J. Geophys. Res., 114, A04211, doi:10.1029/2008JA013912, 2009.

Schriver, D., Ashour-Abdalla, M., Coroniti, F. V., LeBoeuf, J. N., Decyk, V., Travnicek, P., Santolik, O., Winningham, D., Pickett, J. S., Goldstein, M. L., and Fazakerley, V.: Generation of whistler mode emissions in the inner magnetosphere: An event study, J. Geophys. Res., 115, A00F17, doi:10.1029/2009JA014932, 2010.

Su, Z., Gao, Z., Zhu, H., Li, W., Zheng, H., Wang, Y., Wang, S., Spence, H. E, Reeves, G. D., Baker, D. N., Blake, J. B., Funsten, H. O., and Wygant, J. R.: Nonstorm time dropout of radiation belt electron fluxes on 24 September 2013, J. Geophys. Res.-Space, 121, 6400-6416, doi:10.1002/2016JA022546, 2016.

Thorne, R. M., Li, W., Ni, B. , Ma, Q., Bortnik, J., Chen, L., Baker, D. N., Spence, H. E., Reeves, G. D., Henderson, M. G., Kletzing, C. A., Kurth, W. S., Hospodarsky, G. B., Blake, J. B., Fennell, J. F., Claudepierre, S. G., and Kanekal, S. G.: Rapid local acceleration of relativistic radiation-belt electrons by magnetospheric chorus, Nature, 504 , 411-414, doi:10.1038/nature12889, 2013. 
Trakhtengerts, V. Y., Demekhov, A. G., Pasmanik, D. L., Titova, E. E., Kozelov, B. V., Nunn, D., and Rycroft, M. J.: Highly anisotropic distributions of energetic electrons and triggered VLF emissions, Geophys. Res. Lett., 28, 2577-2580, doi:10.1029/2001GL013028, 2001.

Yang, C., Su, Z., Xiao, F., Zheng, H., Wang, Y., Wang, S., Spence, H. E., Reeves, G. D., Baker, D. N., Blake, J. B., and Funsten, H. O.: Rapid flattening of butterfly pitch angle distributions of radiation belt electrons by whistler-mode chorus, Geophys. Res. Lett., 43, 8339-8347, doi:10.1002/2016GL070194, 2016.
Zhelezniakov, V. V. and Zlotnik, E. I.: Cyclotron lines in the spectra of solar flares and solar active regions, Sol. Phys., 121, 449-456, doi:10.1007/BF00161712, 1989. 